\title{
A comprehensive use of ultrasound examination in infertility workup
}

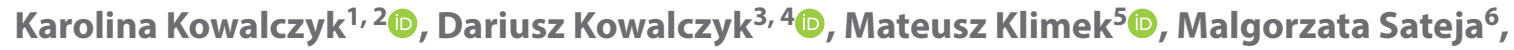

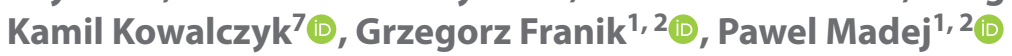 \\ ${ }^{1}$ Department of Gynecological Endocrinology, School of Medicine in Katowice, Medical University of Silesia, Katowice, Poland \\ ${ }^{2}$ Infertility Outpatient Clinic, University Clinical Center, Medical University of Silesia, Katowice, Poland \\ ${ }^{3}$ Department of Anatomy, School of Medicine in Opole, University of Opole, Poland \\ ${ }^{4}$ Department of Gynecology and Obstetrics, Hospital in Nysa, Poland \\ ${ }^{5}$ Department of Gynecology and Obstetrics, School of Medicine in Katowice, Medical University of Silesia in Katowice, Poland \\ ${ }^{6}$ Clinics of Obstetrics and Gynecology, Institute of Mother and Child, Warsaw, Poland \\ ${ }^{7}$ Department of Urology and Urological Oncology, University Hospital in Wroclaw, Poland
}

\begin{abstract}
Considering the growing availability of ultrasound diagnostic methods in gynecology, its role in the infertility setting is increasing. In this review, we present an up-to-date ultrasound based diagnostic scheme in infertility workup comprising the evaluation of ovarian anatomy and function, uterine exploration, as well as tubal patency. The possibility of performing the vast majority of infertility diagnostics by ultrasound in the ambulatory settings is not only attractive and beneficial to patients, but also to health care system. Thus, it is vital for gynecologists to implement modern non-invasive ultrasound modalities in their everyday practice.
\end{abstract}

Key words: infertility; reproduction; ultrasound; antral follicle count; sonohysterography; HyCoSy; HyFoSy; fertility scan

Ginekologia Polska 2021; 92, 6: 453-459

\section{INTRODUCTION}

Imaging diagnostics is the essential part of contemporary medicine. The ultrasound-based examination plays a special role in gynecology and its use has been rising, particularly in infertility workup. Increasingly, ultrasound examinations allow to get a diagnosis without introducing invasive procedure. Patients are properly qualified to have invasive procedures and, owing to modern ultrasound modalities, hysteroscopy and laparoscopy can be applied rather for treatment than simply for diagnosing [1].

In this literature review, we aimed to present an up-to-date ultrasound based diagnostic scheme in infertility workup based on records concerning this field published in English in Pubmed/MEDLINE database from January 2010 to November 2020.

\section{Ultrasound assessment of the ovaries}

Besides hormonal methods, cycle monitoring and counting antral follicles by ultrasound are accepted, complementary methods to evaluate ovarian reserve and function [2]. They are offered to women of reproductive age for various reasons such as in subfertility and ovulatory dysfunction, in infertility and assisted reproduction workup or in predicting the risk of menopause.

The antral follicle count (AFC) includes a total number of antral follicles seen in both ovaries measuring 2 to $10 \mathrm{~mm}$ responsive to follicle-stimulating hormone (FSH) that can be recruited to maturation. Follicles $>10 \mathrm{~mm}$ are referred to as 'dominant' ones. Total AFC (follicles from both ovaries) is used frequently in assisted reproduction centers to predict ovarian response to gonadotropin stimulation, whereas the follicle number per ovary (FNPO) is more useful in gynecological practice to assess functional ovarian reserve [3]. The suggestion of how to interpret follicle count according to Consensus Opinion by Coelho Neto et al. [3] is presented in Table 1.

It has been suggested to examine AFC in the early follicular phase of the menstrual cycle, whereas an ultrasound between 
Table 1. Suggestion of how to interpret follicle count according to Consensus Opinion adapted from Coelho Neto et al. [3]

\begin{tabular}{|l|l|l|}
\hline Nomenclature & Total AFC & Interpretation for ovarian stimulation \\
\hline Very low number of recruitable follicles & $0-4$ & Very high risk of poor response to ovarian stimulation \\
\hline Low number of recruitable follicles & $5-8$ & High risk of poor response to ovarian stimulation \\
\hline Normal number of recruitable follicles & $9-19$ & Normal response to ovarian stimulation expected \\
\hline Large number of recruitable follicles & $\geq 20$ & High risk of excessive ovarian response to ovarian stimulation \\
\hline Nomenclature & FNPO & Interpretation in clinical practice \\
\hline Low follicle count & $1-3$ & Low ovarian reserve \\
\hline Normal follicle count & $4-24$ & Normal ovarian reserve \\
\hline High follicle count & $\geq 25$ & Polycystic pattern \\
\hline
\end{tabular}

AFC — antral follicle count; FNPO — follicle number per ovary

days 10 to 12 determines whether a 'good' dominant follicle is present and shows the endometrial response to follicular development [1]. Scans should be transvaginal (TVS) and with a minimum frequency of $7 \mathrm{MHz}$. Hormonal contraceptives and gonadotropin-releasing hormone agonists may reduce the quantity of follicles seen on an ultrasound, AFC is therefore preferentially measured during a natural cycle or after two to three months without hormone use. The observer-dependence is believed to be a disadvantage of this technique. However, accuracy increases with the operator's skill $[3,4]$.

Both two-dimensional (2D) and three-dimensional (3D) ultrasounds may be employed to perform AFC. On a 2D ultrasound, follicles are counted using either real-time imaging or stored cine-loops. When using a 3D ultrasound, the most common technique is to count the follicles manually in the multi-planar mode. However, there are likewise rendered modes to perform it semi-automatically, for example sonography-based automated volume calculation (sonoAVC ${ }^{\mathrm{TM}}$ by GE Healthcare, United States or syngo ${ }^{\circledR}$ Auto Follicle by Siemens Healthcare $\mathrm{GmbH}$, Erlagen, Germany) [4] (Fig. 1A-B). Further research should include the reproducibility of new volume modalities available for follicle count.

Currently, anti-Mullerian Hormone (AMH) is considered as the most reliable marker for ovarian reserve and it is also recommended by Polish Society of Reproductive Medicine and Embryology (PTMRiE) in basic female fertility assessment [5]. It has been shown that there is a strong positive correlation between serum AMH level and AFC. The use of AMH combined with AFC may improve ovarian reserve evaluation [6].

\section{Ultrasound assessment of the uterine cavity}

The assessment of the uterine cavity is another routine examination performed in patients with subfertility and infertility. In the past, laparoscopy (to assess the outer shape of the uterus) with hysteroscopy (to assess the cavity) were the gold test in diagnosis of congenital uterine anomalies, however, now considering the evidence ultrasound-based techniques seem to play a crucial role [7].
Basic 2D ultrasounds should be employed as a screening tool to assess the uterine cavity and secondary uterine pathologies such as polyps, myomas, uterine adhesions, or adenomyosis [8]. A 2D transvaginal ultrasound performed by an expert in the field, with standardized evaluation of the uterus scans in mid-sagittal and transverse plane, was observed to be highly accurate (84-90.6\%) in the differentiation of arcuate, bicornuate, and septate uteri compared to laparoscopy with hysteroscopy [8]. Compared with conventional 2D ultrasounds, 3D volume imaging has a higher diagnostic accuracy in detecting uterine anomalies (97.1-100\%) [7].

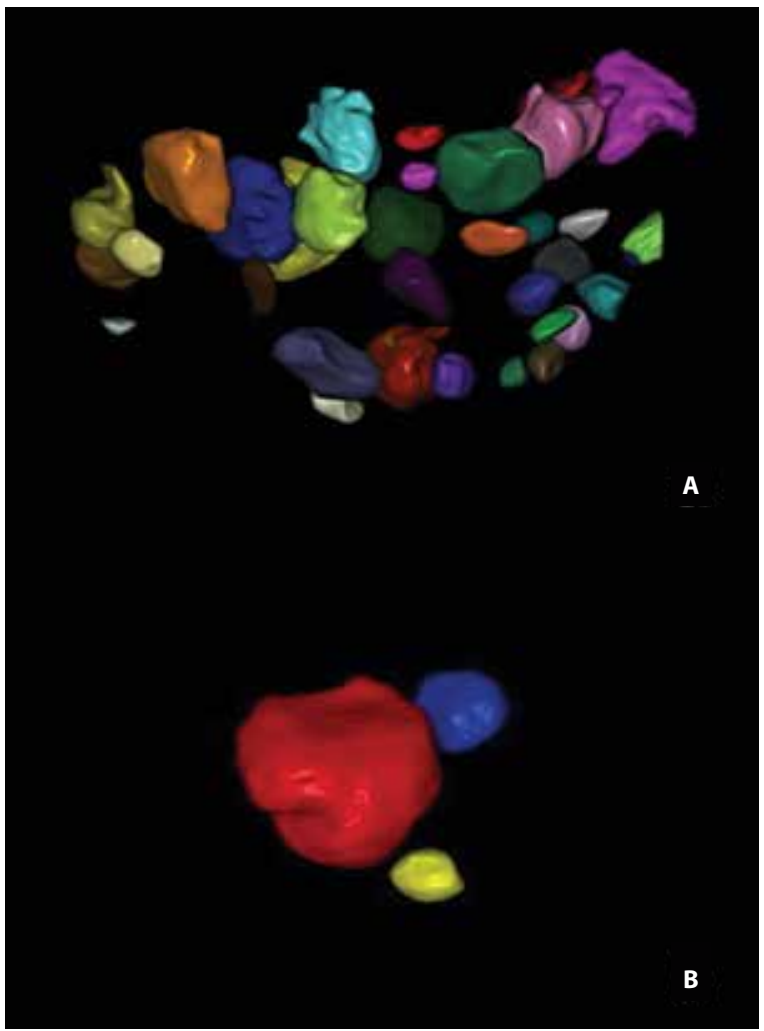

Figure 1. Ovaries visualised with sonoAVC; A. Ovary with several follicles; B. Ovary with few follicles 
Coronal view, which is pivotal for the diagnosis, is rendered from a 3D dataset acquired, either on the ultrasound machine or on the personal computer. The reproducibility of 3D ultrasound is high, that is why it is recommended to be the first diagnostic step in the assessment of the uterine cavity $[8,9]$. To obtain good quality images with clear margins between endometrium and myometrium, the exam should be performed in the second phase of the menstrual cycle [10]. A 3D TVS combined with Power Doppler also has value for the differential diagnosis of endometrial lesions among infertile women. Endometrial thickness and volume were larger among women with endometrial polyps and hyperplasia, whereas endometrial vascularization index, flow index, and vascularization flow index were lower among women with intrauterine adhesions [11].

According to The European Society of Human Reproduction and Embryology (ESHRE) and European Society for Gynaecological Endoscopy (ESGE) consensus on diagnosis of female genital anomalies magnetic resonance imaging (MRI) or eventually endoscopic evaluation are recommended for the subgroup of patients with suspected complex anomalies, diagnostic dilemmas or in case of poor quality of ultrasound visualization [9]. MRI is advised as a first line diagnostic procedure in the case of adolescents [9].

\section{Sonohysterography}

Sonohysterography or saline infusion sonohysterography (SIS) is minimally invasive, outpatient and low-cost method to visualize the endometrial cavity in more detail than is possible with routine transvaginal ultrasounds. Once the uterine cavity is filled with sterile fluid (e.g., saline infusion), a real-time 2D scanning of the uterine cavity is completed. Additional techniques, such as 3D ultrasound, may be used for acquiring coronal view [12] (Fig. 2A). There are also novel, worth mentioning modalities assessing uterine cavity volume and shape using automatic volume calculation software [13] (Fig. 2B). The main indication for 3D-SIS is verification of doubtful 3D-TVS images [8]. Moreover, 2D and 3D-SIS are useful in the diagnosis of diseases closely related to infertility, in particular myomas, endometrial polyps and Asherman's syndrome $[14,15]$. It allows the preoperative evaluation of benign intracavitary lesions [16]. Sonohysterography also plays an important role in secondary fertility investigation due to cesarian scar pregnancy. Recent studies revealed that 3D-SIS is superior in evaluation of the residual myometrial thickness and niche width providing better characterization of the scar niche [17]. It is suggested that uterine niches should always be assessed by SIS, because assessment of niche morphology is commonly dependent on the presence of natural fluids in a niche, which is highly changeable during the menstrual cycle.
Using only 2D TVS may underestimate the prevalence of scar pregnancy defect [18].

According to Ludwin et al. [8], 3D-SIS is the only ultrasound method which provides results consistent with hysteroscopy performed with laparoscopy, considered as the gold standard, in the differential diagnosis of septate, bicornuate and arcuate uteri. SIS showed also significantly higher accuracy (100.0\%) compared to diagnostic hysteroscopy without laparoscopy (80.7\%) in the differential diagnosis of the aforementioned pathologies [19]. It seems that 3D-SIS should be preferred for a final differential diagnosis of the most frequent uterine anomalies if these conditions are not accompanied by other medical indications. The authors emphasize, that hysteroscopy without laparoscopy, which is often performed in these cases, is a suboptimal, poorly reproducible method to differentiate septate and bicornuate uterus, because the outer shape of the uterus cannot be verified [8]. PTMRiE recommends performing diagnostic laparoscopy in patients suspected of pelvic lesions or having risk factors for tubal occlusion, so that the patient could benefit from the surgery [5]. According to Polish Society of Gynecologists and Obstetricians guidelines regarding hysteroscopy, it should be offered routinely in case of intracavitary lesions detected on ultrasound, abnormal uterine bleeding or recurrent miscarriages. As far as uterine cavity assessed on ultrasound is normal, hysteroscopy should not be used as a first line screening tool in infertility workup or before in vitro fertilization procedure [20].

\section{Ultrasound methods of tubal patency investigation}

Hysterosalpingo-contrast-sonography (HyCoSy) was introduced as an alternative to hysterosalpingography (HSG) for outpatient tubal assessment. It overcomes such major drawbacks as hospitalization, radiation exposure and the use of iodinated contrast media.

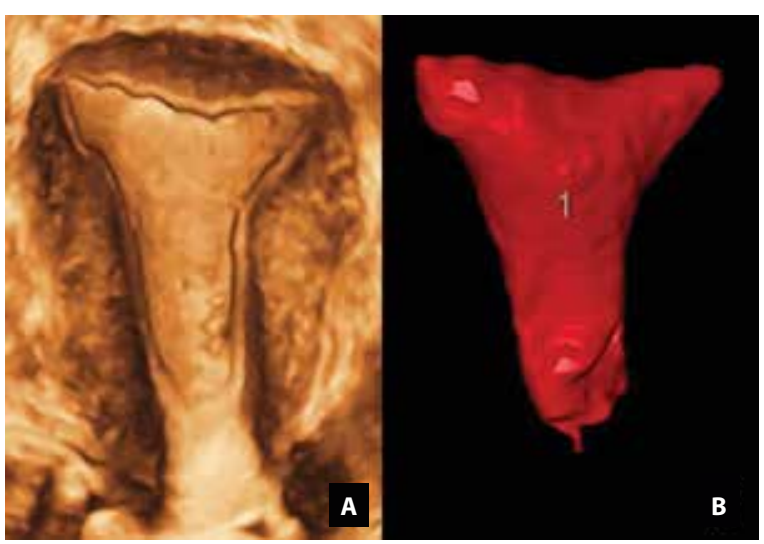

Figure 2. A. 3D sonohysterography; B. Uterine volume estimation using SonoHysteroAVC technique $V=2.5 \mathrm{~mL}$ 
The most accessible contrast is saline, or a mixture of air and saline administered simultaneously or alternately. Subsequently, more hyperechoic contrast media were introduced, e.g., Echovist ${ }^{\oplus}$ (galactose microparticles; Schering, AG, Berlin, Germany), SonoVue ${ }^{\circledast}$ (sulfur hexafluoride; Bracco, Milan, Italy) However, their use is limited because of a high cost or no license for an intrafallopian tube. In 2007, a micro-bubble contrast agent known as ExEm Foam ${ }^{\circledast}$ (GynaecologIQ, Deft, The Netherlands), containing hydroxyethylcellulose and glycerol was launched [21]. Owing to good quality sonograms obtained and acceptable price, hysterosalpingo-foam-sonography (HyFoSy) has become widely adopted in infertility office and ambulatory settings [22].

According to the National Institute for Health and Clinical Excellence (NICE), HyCoSy may be as effective as HSG for diagnosing fallopian tube occlusion, and both appear to have high sensitivity and specificity compared with laparoscopy [23]. NICE, ESHRE, and PTMRiE recommend that it should be offered to women with no comorbidities suggesting pelvic pathology, such as pelvic inflammatory disease, previous ectopic pregnancy or endometriosis [5, 23, 24]. Regarding different contrast media, it seems that high negative predictive value (99.5\%) of air/saline-HyCoSy suggests that this procedure can be implemented as a screening examination. Nevertheless, HyCoSy requires greater experience and is observer-dependent, since the window for visualizing the passage of contrast through the tubes is short [25]. Rapid movements of the probe are necessary for tracing the circuitous or distant tubes in different planes. HyCoSy with more hyperechoic contrasts, e.g., HyFoSy, may be an alternative as the foam fills slowly, the tube and remains stable for at least 5-7 minutes. In addition to this, the use of hyperechoic contrast media does not require a learning period as observed in series for air/saline-HyCoSy [26]. HyFoSy having a significantly higher positive predictive value (30.4\% air/saline $\mathrm{Hy}$ CoSy vs $48 \%$ HyFoSy), is suggested a second-step technique in the event of e.g., inconclusive examination, occlusion suspicion or poor images quality [27]. Additional scanning using high-definition flow Doppler further improves the accuracy of HyFoSy. According to research of Ludwin et al. (2017) [27], it was the only method (with the accuracy of 95.8\%) that did not differ significantly concerning accuracy from laparoscopy with dye chromotubation as the reference method. According to Chinese data 4D-HyCosy represents also highly useful method for diagnosing tubal patency $[28,29]$. Though in recent meta-analysis its diagnostic performance is similar to 2D-HyCoSy [30]. Certainly, future prospective studies comparing both technics in the same set of patients will give more precise answer. Finally, laparoscopy is dedicated to patients when hysterosalpingography remains inconclusive or as a first line diagnostic tool when pelvic pathology influencing the tubes patency is suspected [5].
A tubal patency exam should be carried in the preovulatory phase. The eventual antibiotic prophylaxis is left to examiner's decision as there are still no guidelines, nor randomized controlled trials addressing this issue [31]. HyCoSy was reported to be associated with very low risk of infections. In fact, post-procedural infections were recorded in $0.95 \%$ of patients undergoing $\mathrm{HyCoSy}$ and were absent in HyFoSy studies [32]. An echogenic medium is injected transcervically using a balloon catheter (diameter 5-8 French) or non-balloon dedicated cervical applicator [21]. After confirming the correct placement of the catheter, contrast is slowly injected into the endometrial cavity and in meantime its flow is observed in 2D transverse plane from uterine horn, through each tube until peritoneal spill is visualized (Fig. 3A-B). Additional scanning using high-definition flow Doppler is beneficial, as it improves the accuracy of the exam. The advantage of hyperechoic contrasts is maintaining echogenicity for few minutes; therefore, it allows 3D volume acquisition and visualization of the tubal course in the coronal view (Fig. 4A-B).

In a randomized controlled trial by Dreyer, HyFoSy turned out to be a less painful and less time-consuming tubal patency test compared with HSG [32]. Prophylactic analgesia is unnecessary, however, it is nevertheless considered, indomethacin trans-rectally and paracetamol or codeine orally seems to be effective [33, 34]. Lately, intrauterine lidocaine flushing before HyFoSy has been proven

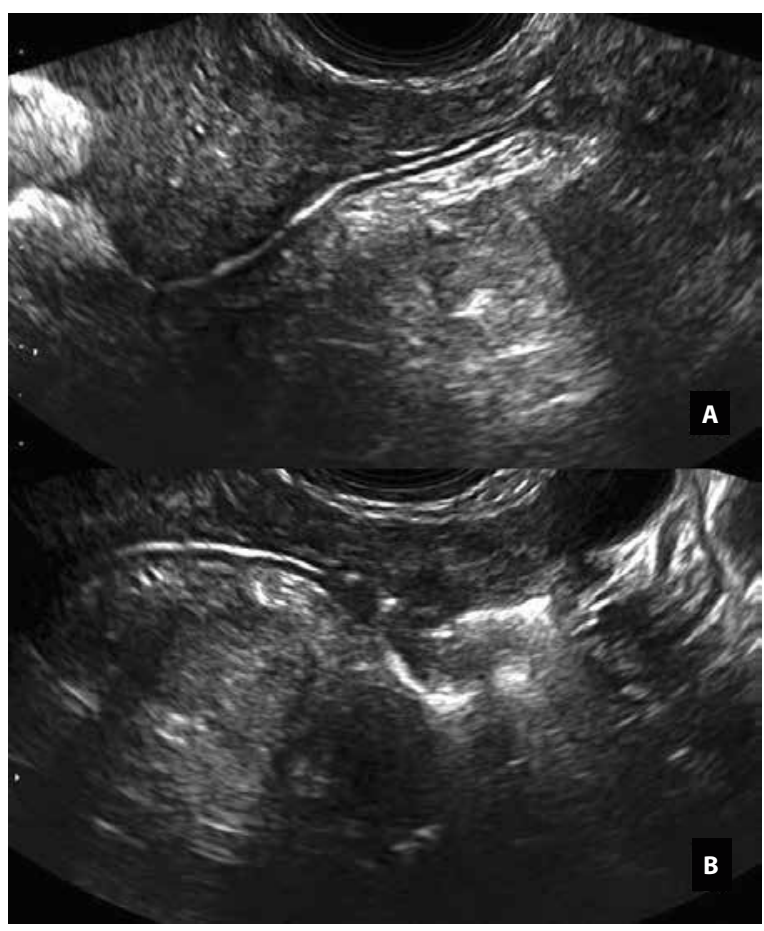

Figure 3. 2D HyFoSy; A. Foam visible in interstitial part, isthmus and ampulla of the left tube; B. Foam visible in infundibulum and then spilling into peritoneum 


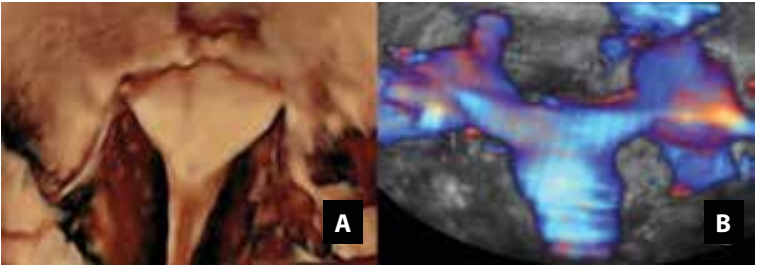

Figure 4. Offline renedered HyFoSy images; A. HD-live 3D HyFoSy; B. HD flow Doppler 3D HyFoSy

to decrease the pain during the procedure [35]. In a study of 632 women who underwent HyCoSy, no late complications were observe. Only $6.48 \%$ of the patient population experienced severe pelvic pain and $4.11 \%$ showed mild vasovagal reactions [36]. Even lower percentage of complications $-0.32 \%$, including vasovagal reactions and mild urinary infection, were reported after HyFoSy. The median visual analogue scale (VAS) score for perception of pain was 2 (range $0-10$ ), 1.9\% of women reported severe pain. Recently, two cases of foam intravasation were reported in the literature [37, 38]. In 2019, U. S. Food and Drug Administration (FDA) approved HyFoSy for the detection of fallopian tube patency in women with known or suspected infertility [39].

So far, only observational studies investigating the chance of subsequent pregnancy after HyCoSy and HyFoSy, are available. Cautious conclusions that fertility enhancing effect may exist were drawn [40]. More is known about the effect of HSG [41]. A recent meta-analysis showed that tubal flushing using oil-based contrast medium compared with water-based contrast medium and no intervention, probably increases clinical pregnancy rates within six months after randomization and may increase subsequent live-birth rates. However, the authors stated that evidence on fertility outcomes beyond sixmonths is inadequate to draw firm conclusions [41]. This year first research on oil-based contrast Lipiodol ${ }^{\circledR}$ (Guerbet LLC, Princeton, New Jersey, USA), previously used in HSG, utilization in HyCoSy was published. According to Zen et al., sonographic visualization of the agitated Lipiodol is similar or better than that of agitated saline [42]. We are waiting for the results of a large, randomized study - the FOAM study, which is currently ongoing. The researchers want to compare the effectiveness and costs of management guided by HyFoSy or by HSG. The primary outcome is ongoing pregnancy leading to live birth within 12 months after randomization [43].

\section{FERTILITY SCAN}

In 2011 Hrehorcak and Nargund described the idea of 'one-stop' fertility assessment [1]. The concept is focused on the investigation both anatomy and function of the ovaries and the uterus, as well as the tubes during one visit in infertility workup between days 10-12 of a regular cycle. To yield the best results the equipment should be of high resolution with sensitive color and spectral Doppler modalities and preferably 3D facilities. It is carried out in one place, saving the couple and the professional valuable time and is $66 \%$ less expensive. It offers a quick, one hour diagnosis in comparison to 18 weeks, on average, of standard multi-visit workup [1]. Lately, fertility scan including sonohysterography and HyFoSy, called Fertiliscan $\odot$, has been proposed [44].

\section{SUMMARY}

A diagnostic strategy has to be safe, noninvasive, well tolerated and possibly at a low cost for the health care system. For these reasons, ultrasound fertility assessment is an accurate choice for the first line in the infertility workup and its use has been rising.

Performing fertility scan, it is advised to count AFC manually using any of the following techniques: real-time 2D TVS, 2D cine-loops, or 3D TVS datasets. The 2D ultrasound is used as a screening tool to assess the uterine cavity. The 3D-TVS and 3D-SIS are recommended as an optimal diagnostic tool in women suspected to have a uterine anomaly. HyCoSy and HyFoSy has proved to be a safe and well tolerated outpatient procedure in the assessment of tubal patency. Air/saline-HyCoSy is considered a screening examination, whereas HyFoSy is suitable for a second-step technique. Further research should concern the diagnostic value of new ultrasound modalities, e.g., 4D-HyCoSy.

The new concepts of 'one-stop' fertility scan, combining all tests in one, seems to be extremely beneficial to infertile couples regarding their fertility potential and stress. One stop fertility diagnosis has high demands on the clinic logistic, high quality ultrasound equipment and clinics ultrasound expertise. Above all, we believe that this is the right direction for the future infertility diagnostics improvement.

\section{Conflict of interest}

The authors declare no conflict of interest.

\section{REFERENCES}

1. Hrehorcak M, Nargund G. "One-Stop" fertility assessment using advanced ultrasound. Facts Views Vis Obgyn. 2011; 3(1): 8-12, indexed in Pubmed: 24753842

2. Committee opinion no. 618: Ovarian reserve testing. Obstet Gynecol 2015; 125(1): 268-273, doi: 10.1097/01.AOG.0000459864.68372.ec, indexed in Pubmed: 25560143.

3. Coelho Neto MA, Ludwin A, Borrell A, et al. Counting ovarian antral follicles by ultrasound: a practical guide. Ultrasound Obstet Gynecol. 2018; 51(1): 10-20, doi: 10.1002/uog.18945, indexed in Pubmed: 29080259.

4. ReC, Mignini Renzini M, Rodriguez A, et al. From a circle to a sphere: the ultrasound imaging of ovarian follicle with 2D and 3D technology. Gynecol Endocrinol. 2019; 35(3): 184-189, doi: 10.1080/09513590.2018.1522297, indexed in Pubmed: 30394144. 
5. Łukaszuk K, Kozioł K, Jakiel G, et al. Diagnostyka i leczenie niepłodności — rekomendacje Polskiego Towarzystwa Medycyny Rozrodu i Embriologii (PTMRiE) oraz Polskiego Towarzystwa Ginekologów i Położników (PTGP). Ginekol Perinatol Prakt. 2018; 3(3): 112-140.

6. Iliodromiti S, Anderson RA, Nelson SM. Technical and performance characteristics of anti-Müllerian hormone and antral follicle count as biomarkers of ovarian response. Hum Reprod Update. 2015; 21(6): 698-710, doi:10.1093/humupd/dmu062, indexed in Pubmed: 25489055.

7. Chan YY, Jayaprakasan K, Zamora J, et al. The prevalence of congenital uterine anomalies in unselected and high-risk populations: a systematic review. Hum Reprod Update. 2011; 17(6): 761-771, doi: 10.1093/humupd/dmr028, indexed in Pubmed: 21705770.

8. Ludwin A, Pityński K, Ludwin I, et al. Two- and three-dimensional ultrasonography and sonohysterography versus hysteroscopy with laparoscopy in the differential diagnosis of septate, bicornuate, and arcuate uteri. J Minim Invasive Gynecol. 2013; 20(1): 90-99, doi: 10.1016/j. jmig.2012.09.011, indexed in Pubmed: 23312248.

9. Grimbizis GF, Di Spiezio Sardo A, Saravelos SH, et al. The Thessaloniki ESHRE/ESGE consensus on diagnosis of female genital anomalies. Hum Reprod. 2016; 31(1): 2-7, doi: 10.1093/humrep/dev264, indexed in Pubmed: 26537921

10. Benacerraf $B$. Three-dimensional volume imaging in gynecology. Obstet Gynecol Clin North Am. 2019; 46(4): 755-781, doi: 10.1016/j. ogc.2019.07.008.

11. Ni J, Han B, Liang J, et al. Three-dimensional 3D ultrasound combined with power Doppler for the differential diagnosis of endometrial lesions among infertile women. Int J Gynaecol Obstet. 2019; 145(2): 212-218, doi: 10.1002/ijgo.12787, indexed in Pubmed: 30763458.

12. Technology assessment no. 12: sonohysterography. Obstet Gynecol. 2016; 128(2): e38-e42, doi: 10.1097/aog.0000000000001588, indexed in Pubmed: 27454735.

13. Ludwin A, Martins WP, Ludwin I. Uterine cavity imaging, volume estimation and quantification of degree of deformity using automatic volume calculation: description of technique. Ultrasound Obstet Gynecol. 2017; 50(1): 138-140, doi: 10.1002/uog.15890, indexed in Pubmed: 26919726.

14. Seshadri S, El-Toukhy T, Douiri A, et al. Diagnostic accuracy of saline infusion sonography in the evaluation of uterine cavity abnormalities prior to assisted reproductive techniques: a systematic review and meta-analyses. Hum Reprod Update. 2015; 21(2): 262-274, doi: 10.1093/humupd/dmu057, indexed in Pubmed: 25505226.

15. Anioł M, Dec G, Wojda K, et al. Usefulness of saline infusion sonohysterography and feeding artery imaging in endometrial polyp diagnosis. Ginekol Pol. 2017; 88(6): 285-288, doi: 10.5603/GP.a2017.0054, indexed in Pubmed: 28727125.

16. Nieuwenhuis LL, Hermans FJr, Bij de Vaate AJ, et al. Three-dimensional saline infusion sonography compared to two-dimensional saline infusion sonography for the diagnosis of focal intracavitary lesions. Cochrane Database Syst Rev. 2017; 5(5): CD011126, doi: 10.1002/14651858. CD011126.pub2, indexed in Pubmed: 28472862.

17. Alalfy $M$, Osman OM, Salama $\mathrm{S}$, et al. Evaluation of the cesarean scar niche in women with secondary infertility undergoing ICSI using 2D sonohysterography versus 3D sonohysterography and setting a standard criteria; alalfy simple rules for scar assessment by ultrasound to prevent health problems for women. Int J Womens Health. 2020; 12: 965-974, doi: 10.2147/IJWH.S267691, indexed in Pubmed: 33177887.

18. Antila-Långsjö R, Mäenpää JU, Huhtala H, et al. Comparison of transvaginal ultrasound and saline contrast sonohysterography in evaluation of cesarean scar defect: a prospective cohort study. Acta Obstet Gynecol Scand. 2018; 97(9): 1130-1136, doi: 10.1111/aogs.13367, indexed in Pubmed: 29754409.

19. Ludwin A, Ludwin I, Banas T, et al. Diagnostic accuracy of sonohysterography, hysterosalpingography and diagnostic hysteroscopy in diagnosis of arcuate, septate and bicornuate uterus. J Obstet Gynaecol Res. 2011; 37(3): 178-186, doi: 10.1111/j.1447-0756.2010.01304.x, indexed in Pubmed: 21314802

20. Zimmer M, Pomorski M, Kamiński P, et al. Polish Society of Gynecologists and Obstetricians Guidelines for the application of hysteroscopy in gynecology. Ginekol Pol. 2019; 90(8): 482-489, doi: 10.5603/GP.2019.0083, indexed in Pubmed: 31482553.

21. Emanuel MH, Exalto N. Hysterosalpingo-foam sonography (HyFoSy): a new technique to visualize tubal patency. Ultrasound Obstet Gynecol. 2011; 37(4): 498-499, doi: 10.1002/uog.8912, indexed in Pubmed: 21433170.
22. Exalto N, Emanuel MH. Clinical aspects of HyFoSy as tubal patency test in subfertility workup. Biomed Res Int. 2019; 2019: 4827376, doi: 10.1155/2019/4827376, indexed in Pubmed: 31360713.

23. O'Flynn N. Assessment and treatment for people with fertility problems: NICE guideline. Br J Gen Pract. 2014; 64(618): 50-51, doi: 10.3399/bjgp14X676609, indexed in Pubmed: 24567574.

24. Strowitzki T. Gute Klinische Behandlung ("Good clinical treatment ${ }^{\prime \prime}$ ) bei Maßnahmen der assistierten Reproduktion (ART). Gynäkologische Endokrinologie. 2009; 7(1): 39-44, doi: 10.1007/s10304-008-0301-6.

25. Engels V, Medina M, Antolín E, et al. Feasibility, tolerability, and safety of hysterosalpingo-foam sonography (hyfosy). multicenter, prospective Spanish study. J Gynecol Obstet Hum Reprod. 2021; 50(5): 102004, doi: 10.1016/j.jogoh.2020.102004, indexed in Pubmed: 33242678.

26. Mandia L, Personeni $C$, Antonazzo $P$, et al. Ultrasound in infertility setting: optimal strategy to evaluate the assessment of tubal patency. Biomed Res Int. 2017; 2017: 3205895, doi: 10.1155/2017/3205895, indexed in Pubmed: 29376069.

27. Ludwin I, Ludwin A, Wiechec $M$, et al. Accuracy of hysterosalpingo-foam sonography in comparison to hysterosalpingo-contrast sonography with air/saline and to laparoscopy with dye. Hum Reprod. 2017; 32(4): 758-769, doi: 10.1093/humrep/dex013, indexed in Pubmed: 28184447.

28. Chen F, Quan J, Huang P, et al. Hysterosalpingo-contrast sonography with four-dimensional technique for screening fallopian tubal patency: let's make an exploration. J Minim Invasive Gynecol. 2017; 24(3): 407-414, doi: 10.1016/j.jmig.2016.12.011, indexed in Pubmed: 28034794.

29. Gao YB, Yan JH, Yang YD, et al. Diagnostic value of transvaginal four-dimensional hysterosalpingo-contrast sonography combined with recanalization in patients with tubal infertility. Niger J Clin Pract. 2019;22(1): 46-50, doi: 10.4103/njcp.njcp_376_17, indexed in Pubmed: 30666019.

30. Alcázar JL, Martinez A, Duarte M, et al. Two-dimensional hysterosalpingo-contrast-sonography compared to three/four-dimensional hysterosalpingo-contrast-sonography for the assessment of tubal occlusion in women with infertility/subfertility: a systematic review with meta-analysis. Hum Fertil (Camb). 2020 [Epub ahead of print]: 1-13, doi: 10.1080/14647273.2020.1769204, indexed in Pubmed: 32484066.

31. Thinkhamrop J, Laopaiboon M, Lumbiganon P. Prophylactic antibiotics for transcervical intrauterine procedures. Cochrane Database Syst Rev. 2007; 3: CD005637, doi: 10.1002/14651858.CD005637.pub2, indexed in Pubmed: 17636811.

32. Dreyer K, Out R, Hompes PGA, et al. Hysterosalpingo-foam sonography, a less painful procedure for tubal patency testing during fertility workup compared with (serial) hysterosalpingography: a randomized controlled trial. Fertil Steril. 2014; 102(3): 821-825, doi: 10.1016/j.fertnstert.2014.05.042, indexed in Pubmed: 24996498.

33. Karaman E, Çim N, Alkış I, et al. Rectal indomethacin use in pain relief during hysterosalpingography: A randomized placebo controlled trial. J Obstet Gynaecol Res. 2016; 42(2): 195-201, doi: 10.1111/jog.12863, indexed in Pubmed: 26711715.

34. Ludwin I, Martins WP, Nastri CO, et al. Pain intensity during ultrasound assessment of uterine cavity and tubal patency with and without painkillers: prospective observational study. J Minim Invasive Gynecol. 2017; 24(4): 599-608, doi: 10.1016/j.jmig.2017.01.015, indexed in Pubmed: 28159714.

35. Melcer $Y$, Nimrodi M, Levinsohn-Tavor O, et al. Analgesic efficacy of intrauterine lidocaine flushing in hysterosalpingo-foam sonography: a double-blind randomized controlled trial. J Minim Invasive Gynecol. 2020 [Epub ahead of print], doi: 10.1016/j.jmig.2020.11.019, indexed in Pubmed: 33249268.

36. Marci R, Marcucci I, Marcucci AA, et al. Hysterosalpingocontrast sonography (HyCoSy): evaluation of the pain perception, side effects and complications. BMC Med Imaging. 2013; 13: 28, doi: 10.1186/1471-234213-28, indexed in Pubmed: 23968513.

37. Ludwin A, Ludwin I, Martins WP. Venous intravasation during evaluation of tubal patency by ultrasound contrast imaging. Ultrasound Obstet Gynecol. 2018; 51(1): 143-145, doi: 10.1002/uog.17405, indexed in Pubmed: 28078761.

38. Ludwin A, Ludwin I, Szczeklik W, et al. Cutaneous small-vessel vasculitis following hysterosalpingo-foam sonography (HyFoSy). Ultrasound Obstet Gynecol. 2019; 54(6): 831-834, doi: 10.1002/uog.20372, indexed in Pubmed: 31219636.

39. Drugs@FDA: FDA-Approved Drugs. https://www.accessdata.fda. gov/drugsatfda_docs/label/2020/125514s088lbl.pdf (2020-11-04).

40. Tanaka K, Chua J, Cincotta $R$, et al. Hysterosalpingo-foam sonography (HyFoSy): Tolerability, safety and the occurrence of pregnancy 
post-procedure. Aust N Z J Obstet Gynaecol. 2018; 58(1): 114-118, doi: 10.1111/ajo.12716, indexed in Pubmed: 28949014.

41. Wang $R$, van Welie $N$, van Rijswijk J, et al. Effectiveness on fertility outcome of tubal flushing with different contrast media: systematic review and network meta-analysis. Ultrasound Obstet Gynecol. 2019; 54(2): 172-181, doi: 10.1002/uog.20238, indexed in Pubmed: 30740799.

42. Zen $M$, Kirby A, Dowthwaite $S$, et al. Lipiodol visibility under ultrasound. Aust N Z J Obstet Gynaecol. 2020; 60(4):598-604, doi: 10.1111/ajo.13150, indexed in Pubmed: 32207160.
43. van Rijswijk J, van Welie N, Dreyer K, et al. The FOAM study: is Hysterosalpingo foam sonography (HyFoSy) a cost-effective alternative for hysterosalpingography (HSG) in assessing tubal patency in subfertile women? Study protocol for a randomized controlled trial. BMC Womens Health. 2018; 18(1):64, doi: 10.1186/s12905-018-0556-6, indexed in Pubmed: 29743106.

44. Levaillant JM, Pasquier M, Massin N. A novel concept for female infertility exploration: the Fertiliscan $\odot$, a dedicated all-in-one 3D ultrasound exploration. J Gynecol Obstet Hum Reprod. 2019; 48(5): 363-367, doi: 10.1016/j.jogoh.2019.01.008, indexed in Pubmed: 30690086. 